\title{
Reactive arthritis associated with L2b lymphogranuloma venereum proctitis
}

Khalil El Karoui $^{1,2}$, Frédéric Méchaï ${ }^{1,2}$, Florence Ribadeau-Dumas ${ }^{1,2}$, Jean-Paul Viard ${ }^{1,2}$, Marc Lecuit $^{1,2}$, Bertille de Barbeyrac ${ }^{3}$, Olivier Lortholary ${ }^{1,2} *$

1 Centre d'Infectiologie Necker Pasteur

2 Université Paris Descartes, Service des Maladies Infectieuses et Tropicales, Hôpital Necker Enfants-Malades, Paris, France

3 Centre National de Référence des Infections à Chlamydiae, Laboratoire de Bactériologie, Université Victor Segalen, Bordeaux, France

* to whom correspondance should be adressed

Prof. O. Lortholary

Service des Maladies Infectieuses et Tropicales, Hôpital Necker Enfants-Malades 149 rue de Sèvres 75015 Paris

Phone: 33142192663

Fax: 33142192622

olivier.lortholary@nck.aphp.fr

Key words: Chlamydia trachomatis; lymphogranuloma venereum; reactive arthritis; HIV infection

Word count: 750

Abstract word count: 73 
The Corresponding Author has the right to grant on behalf of all authors and does grant on behalf of all authors, an exclusive licence (or non exclusive for government employees) on a worldwide basis to the BMJ Publishing Group Ltd to permit this article (if accepted) to be published in STI and any other The Corresponding Author has the right to grant on behalf of all authors and does grant on behalf of all authors, an exclusive licence (or non exclusive for government employees) on a worldwide basis to the BMJ Publishing Group Ltd to permit this article (if accepted) to be published in STI and any other BMJPGL products and sub-licences such use and exploit all subsidiary rights, as set out in our licence http://sti.bmijournals.com/ifora/licence.pdf)

\section{Authors contributions:}

K.E.K, F.M., and O.L. were in charge of the patient, and wrote the manuscript.

F.R.D, J.P.V, and M.L. were in charge of the patient, and carefully revised the manuscript.

B.de B. performed the strain serovar identification and carefully revised the manuscript. 


\section{Abstract}

An ongoing outbreak of lymphogranuloma venereum (LGV) L2b proctitis, predominantly in human immunodeficiency virus (HIV)-positive men who have sex with men (MSM) has been reported in industrialized countries. We describe a case of reactive arthritis after L2b proctitis. This case expands the spectrum of severe complications related to LGV L2b proctitis. Since this infection may be asymptomatic, this organism should be screened for in HIV positive MSM with symptoms consistent with reactive arthritis. 


\section{INTRODUCTION}

Chlamydia trachomatis (CT) is the etiological agent of major human infections including trachoma (classically serovars A-C), urogenital infections (serovars D-K) and lymphogranuloma venereum (LGV) (serovars L1-L3).

Since 2003, an ongoing LGV proctitis outbreak has been reported in industrialized countries, including France (mainly in Paris).[1] To date, most LGV isolates of the recent outbreak have been identified as L2b.[2] We report a case of a sexually acquired reactive arthritis (SARA) associated with a CT serovar L2b proctitis in an HIV-infected man.

\section{CASE REPORT}

A 41-year-old Caucasian homosexual man was admitted in our infectious diseases department for oligoarthritis. HIV-1 infection had been diagnosed 4 years earlier, when he presented with cutaneous secondary syphilis. Antiretroviral therapy had not yet been commenced as he had remained well.

One month after an episode of unprotected anal receptive intercourse, he presented with weight loss, fever, purulent rectal discharge, and tenesmus. Proctitis was clinically diagnosed, and he was successfully treated with oral doxycycline (100 mg twice/day). Polymerase chain reaction (PCR) for Chlamydia trachomatis $(\mathrm{CT})$ was positive on taken blind anal sample. The LGV variant L2b was detected by genotyping (PCR-RFLP) of the omp 1 gene encoding the major outer membrane protein (MOMP) and sequencing the variable domain 2 of omp 1 gene. [3]Seven days after the diagnosis of proctitis, he presented with bilateral serous conjunctivitis. A few days later, the patient was hospitalized for investigation of oligoarthritis of the right wrist, right knee and both ankles. Physical examination revealed pain and swelling of right wrist, right knee, both ankles and left metatarso-phalangeal joints, with sausaging of second and third left toes. Laboratory tests were as follows: CD4 lymphocyte count $252 / \mu \mathrm{L}(21 \%)$, 
HIV-1 viral load 6200 genome copies/mL (Log 3.8), negative serological tests for Yersinia, Salmonella, and Campylobacter, negative stool culture. HLA B27 testing was positive while search for rheumatoid factor, antinuclear antibodies and anti-cyclic citrullinated peptide antibodies was negative. Analysis of right knee synovial fluid revealed a raised white blood cells of 4900/mL (90\% neutrophils) and negative bacterial culture. 16S rRNA gene PCR amplification and CT-specific PCR on synovial fluid were both negative. Cultures for $N$. gonorrhoeae, and Mycoplasma species from urethral swab were negative, as well as CTspecific PCR on first void urine sample. X-ray examinations of all involved joints revealed no abnormality.

A diagnosis of SARA complicating CT L2b proctitis was proposed. Oral doxycycline was continued for a total of 30 days (200mg/day). Oral ketoprofen $(150 \mathrm{mg} /$ day) allowed complete clinical recovery after 2 months, and no relapse occurred after one year of follow-up.

\section{DISCUSSION}

Our patient presented with a SARA, 2 weeks after a CT serovar L2b infectious proctitis. Of note, conjunctivis was bilateral and resolved spontaneously, which argues against a direct conjunctive CT infection. Other classic features of SARA, such as balanitis, or sacro-ileitis were absent. However, our patient fulfilled the currently proposed criteria for reactive arthritis $(\operatorname{ReA}) \cdot[4]$

The association of urogenital CT (serovars D-K) with SARA is well described. [5, 6] This association, and the strong temporal relationship between SARA and proctitis in our patient argues for a SARA related to CT L2b proctitis. Cases of ReA associated proctitis and CT infection have already been published, but direct evidence of CT infection was not available, co-infection with other ReA associated-microorganisms was not ruled out, and serovar was not determined.[7, 8] 
Differential diagnoses, including $(i)$ infection with other ReA triggering microorganisms and (ii) gonococcal arthritis, were ruled out with (i) serological tests, cultures of urethral swab, and (ii) 16S rRNA gene PCR on synovial fluid. ReA has been reported to occur in up to $10 \%$ patients with HIV infection. However, there is no direct correlation between ReA and HIV infection per se, and this association is thought to be related to the co-infections occurring in HIV-infected patients, as shown in our case.[9]

In the recent outbreak, LGV predominantly caused proctitis or proctocolitis, primarily through unprotected anal receptive or insertive intercourse,[1] a substantial proportion of patients even being asymptomatic.[10] Diagnosis is essential as prolonged treatment (3 weeks) with doxycycline or macrolides is required in $\mathrm{LGV}$ (CT serovar L1-3) proctitis, in contrast to infection with other serovars for which short term treatment is possible.[10]

Given the recent outbreak, CT L2b proctitis associated SARA appears to be rare, and less frequent than SARA related to other CT serovars (incidence estimated around 1-3\% in CT DK serovars).[4] Strong local immune response in proctitis could prevent dissemination of infection and subsequent ReA.[4]

In conclusion, SARA may be triggered by epidemic LGV L2b isolate responsible for proctitis in HIV-infected MSM.

Word count : 750

Abstract word count: 73 
CONFLICT OF INTEREST. None

ACKNOWLEDGEMENTS. The authors thank Maithe Clerc for genotyping analysis and Nicolas Dupin for his careful review of the manuscript.

\section{KEY MESSAGES}

1- LGV L2b, as well as other CT serovars, may trigger SARA in HLAB27-positive HIVinfected men who have sex with men.

2- Given the recent outbreak, LGV L2b infection should be systematically screened for in MSM with reactive arthritis, even though no proctitis symptoms are present.

3- This case adds reactive arthritis to the severe complications of LGV L2b proctitis, and underscores the importance of rapidly diagnose and treat this infection. 


\section{REFERENCES}

1. Ward H, Martin I, Macdonald N, et al. Lymphogranuloma venereum in the United kingdom. Clin Infect Dis 2007; 44 :26-32.

2. Spaargaren J, Fennema HS, Morre SA, et al. New lymphogranuloma venereum Chlamydia trachomatis variant, Amsterdam. Emerg Infect Dis 2005; 11:1090-2.

3. Rodriguez P, Vekris A, de Barbeyrac B, et al. Typing of Chlamydia trachomatis by restriction endonuclease analysis of the amplified major outer membrane protein gene. $J$ Clin Microbiol 1991; 29:1132-6.

4. Rihl M, Klos A, Kohler L, et al. Infection and musculoskeletal conditions: Reactive arthritis. Best Pract Res Clin Rheumatol 2006; 20:1119-37.

5. Keat A, Thomas B, Dixey J, et al. Chlamydia trachomatis and reactive arthritis: the missing link. Lancet 1987; 1:72-4.

6. Butrimiene I, Ranceva J, Griskevicius A. Potential triggering infections of reactive arthritis. Scand J Rheumatol 2006; 35:459-62.

7. Keat A, Thomas BJ, Taylor-Robinson D. Chlamydial infection in the aetiology of arthritis. Br Med Bull 1983; 39:168-74.

8. Neumann S, Kreth F, Schubert S, et al. Reiter's syndrome as a manifestation of an immune reconstitution syndrome in an HIV-infected patient: successful treatment with doxycycline. Clin Infect Dis 2003; 36:1628-9.

9. Reveille JD, Williams FM. Infection and musculoskeletal conditions: Rheumatologic complications of HIV infection. Best Pract Res Clin Rheumatol 2006; 20:1159-79.

10. Van der Bij AK, Spaargaren J, Morre SA, et al. Diagnostic and clinical implications of anorectal lymphogranuloma venereum in men who have sex with men: a retrospective case-control study. Clin Infect Dis 2006; 42:186-94. 